\title{
La resección transuretral de la próstata de baja presión hidráulica, resultados en 340 pacientes con adenomas grandes
}

\author{
Verger-Kuhnke AB, Reuter MA, Epple W, Ungemach G. \\ Reuter Klink, Servicio de Urología. Hospital Karl-Olga, Hospital Escuela Dependiente de la Universidad de \\ Ulm, Stuttgart Alemania.
}

Actas Urol Esp. 2006;30(9):896-904

\begin{abstract}
RESUMEN
LA RESECCIÓN TRANSURETRAL DE LA PRÓSTATA DE BAJA PRESIÓN HIDRÁULICA, RESULTADOS EN 340 PACIENTES CON ADENOMAS GRANDES

Introducción: La resección transuretral de próstata (RTUP) es el tratamiento electivo para los adenomas pequeños y medianos. En este trabajo se analizan la efectividad, los resultados y la morbilidad peri-operatoria en la resección de los adenomas grandes.

Pacientes y método: Se analizan los resultados de 340 pacientes con adenomas grandes y obstucción infravesical sintomática que fueron operados con la RTUP de baja presión hidráulica entre agosto de 1999 y junio del 2006.

Resultados: Edad promedio de los pacientes 69 años (rango 51-89). Volumen prostático por TRUS, 107 ml (70-204). PSA 7,94ng/ml (0,71-26,4). Peso de los fragmentos resecados: 74,5gr (50-160), tiempo de RTUP 65 min. (35-155), sondaje uretral postoperatorio 1,7 dias (1-8), sondaje suprapúbico 6,5 días (5-15), estancia hospitalaria 8 dias (7-16), flujometria máx. preoperatoria 11,2 ml/seg. (5-15,7), postoperatoria 19,7 ml/seg. (7-41,3). En ningún caso de este grupo se observó el síndrome de RTUP.

Complicaciones operatorias: Infección urinaria sin fiebre (Bac. >100.000) 95 pac. (27,9\%), sondaje vesical por retención urinaria pre-operatoria 53 pac. (15,6\%), retención urinaria post-operatoria 18 pac. $(5,3 \%)$, infección urinaria con fiebre $>38^{\circ} \mathrm{C} 11$ pac. $(3,2 \%)$, tamponamiento vesical por sangrado post-operatorio 7 pac.(2\%), falsa vía en 2 pac. (0,5\%), hematoma escrotal masivo (luego de vasectomia) en 1 pac. (0,3\%).

Control de la hemoglobina pre y postoperatoria: Hb pre-op 15 g/dl (12-19,3), Hb post-op 11,5 g/dl (7,6-16,4), diferencia (- 3,5g/dl) $23,3 \%$.

En sólo 29 pacientes $(8,5 \%)$ fue necesario efectuar una transfusión sanguínea (heteróloga) de 2 a 4 bolsas (500ml c/u), la TRUS fue de $125 \mathrm{ml}$, el tejido resecado $90 \mathrm{~g}$ (52-140), la Hb preoperatoria fue de 14,72g/dl y la post-op de 8,8g/dl con una diferencia de (-5,92g/dl) $40,2 \%$.

Conclusiones: La RTUP vídeo-asistida de baja presión hidráulica, es un método efectivo en el tratamiento quirúrgico en adenomas prostáticos grandes. Las complicaciones postoperatorias severas son escasas, y en 311 pacientes (91,5\%) no fue necesaria ninguna transfusión sanguínea. La estancia hospitalaria así como el sondaje uretral son menores en comparación con la adenomectomía abierta.
\end{abstract}

Palabras claves: RTUP de baja presión hidráulica. HPB. Adenomas grandes. Hemoglobina.

\section{ABSTRACT}

THE LOW HYDRAULIC PRESSURE TRANSURETHRAL RESECTION OF THE PROSTATE RESULTS IN 340 PATIENTS WITH GREAT ADENOMAS Introduction: the transurethral resection of prostate TURP is the elective treatment for the small and medium adenomas. In this study they analyse the effectiveness, the results and the postoperative morbidity in the resection of the great adenomas.

Patients and Method: We study 340 patients with great adenomas and symptomatic infravesical obstruction that were operated with the low hydraulic pressure RTUP between August of 1999 and June of 2006.

Results: Average Age of the patients 69 years (range 51-89). Prostate volume by TRUS, $107 \mathrm{ml}(70-204)$. PSA 7,94 ng/ml (0,71-26,4). Weight of the resected fragments: 74,5gr. (50-160), time of the intervention 65 min. (35-155), postoperative urethral catheterisation 1.7 days (1-8), suprapubic derivation 6.5 days (5-15), the duration of hospital stay after surgery were 8 days (7-16), peak flow pre-op.11.2 $\mathrm{ml} / \mathrm{sec}$. (5-15,7), post-operative $19.7 \mathrm{ml} / \mathrm{sec}$ (7-41,3). There were no cases of TURP syndrome in this group.

Operative complications: Urinary infection without fever (bacteria $>100.000) 95$ patients $(27.9 \%)$, bladder derivation by preoperative urinary retention 53 patients (15,6\%). 18 patients $(5,3 \%)$ with postoperative urinary retention. 11 patients $(3,2 \%)$, with infection and fever $>38^{\circ} \mathrm{C}$. 7 patients $(2 \%)$ with postoperative bladder bleeding, 2 patients $(0,6 \%)$ with urethral lesion. 1 patient $(0.3 \%)$, with massive scrotal hematoma (after vasectomy).

Control of the pre and post operative hemoglobin: Hemoglobin pre-op $15 \mathrm{~g} / \mathrm{dl}(12-19,3)$, hemoglobin post-op 11,5 g/dl (7,6-16,4), difference of $(-3,5 \mathrm{~g} / \mathrm{dl}) 23,3 \%$. In only 29 patients $(8.5 \%)$ it was necessary to carry out a sanguineous transfusions (heterolog) of 2 to 4 EC (500ml), the TRUS was $125 \mathrm{ml}$, weight of the fragments 90 gr. (52-140), the hemoglobin pre-op was 14,72g/dl and post-op of $8,8 \mathrm{~g} / \mathrm{dl}$ with a difference of $(-5,92 \mathrm{~g} / \mathrm{dl}) 40.2 \%$.

Conclusions: The video assisted low hydraulic pressure TURP, is an effective method in the surgical treatment of great prostate adenomas. The severe postoperative complications are little, and in 311 patients (91.5\%) it was not necessary sanguineous transfusion. The stationary treatment and the urethral catheterisation is smaller in comparison with the open adenomectomy.

Keywords: Low hydraulic pressure TURP. BPH. Great adenomas. Hemoglobin. 
$\mathrm{E}^{1}$ interés de observar el interior de la vejiga existe desde tiempos inmemoriales, pero a diferencia del intestino, vagina o cavidad bucal, donde existían instrumentos para su inspección hace cientos de años, fue a mediados del siglo XIX cuando se comenzó con la fabricación de un instrumento para la exploración vesical.

Esto se basa en el fenómeno de que la palpación vesical "Tactus eruditus", tiene una larga tradición en Urología, así desde la antigüedad y con la ayuda de sondas uretrales se podían diagnosticar cálculos vesicales o determinar el crecimiento prostático intravesical y debido a esto, durante mucho tiempo no se produjeron avances significativos en otros métodos diagnósticos por no creerlos necesarios ${ }^{1}$.

En este largo proceso que llevó a la invención del cistoscopio intervinieron muchos médicos y técnicos que intentaron unificar en un instrumento los 3 componentes básicos de un endoscopio como son: la sonda, el medio conductor de la luz y la fuente de luz misma.

Maximilian Nitze (1848-1906) trabajaba en 1876 como médico residente en ginecología en Dresden, Alemania, y fue allí donde comenzó con la fabricación de su instrumento que denominó “cistoscopio". Él es el primero que traslada la fuente lumínica al extremo distal del instrumento y así al interior de la vejiga, logran su mejor visibilidad. El 2 de Octubre de 1877, ante la real Sociedad de Medicina en Dresden efectuó una uretro-cistoscopía en un cadáver al cual se le había introducido previamente una piedra vesical, la cual se pudo reconocer ${ }^{1}$.

La invención del cistoscopio permitió la intervención quirúrgica transuretral bajo visión directa.

Al principio se resecaban cantidades pequeñas de tejido, pero a partir de la década de 1940, con la mejora de los sistemas ópticos y técnicos se permitían resecciones de hasta $30 \mathrm{~g}$. A partir de de entonces es cuando se pudo comparar este método endoscópico con la cirugía abierta y convertirse en el procedimiento de elección en el tratamieneto de la HPB obstructi$\mathrm{va}^{1,2}$.

La resección transuretral de próstata (RTUP) desde hace varios años es el tratamiento electivo, el "Standard gold" para los adenomas de pequeño y mediano tamaño ${ }^{3-5}$.
Sobre todo la hemorragia peri-operatoria y el síndrome de RTU han sido el motivo para la introducción de otras técnicas quirúrgicas menos invasivas con la intención de reducir dichas complicaciones.

Los otros tratamientos disponibles como el Holmium láser, Nd:Yag láser, el KTP-láser de 2040 y 80-120 watt, la adenomectomía laparoscópica, la resección bipolar, la resección en medio salino, el rotorresector, asi como los procedimientos abiertos, como la operación de Freyer y Millin con sus modificaciones, son opciones alternativas a las RTUP3,6-18.

En un futuro se verá si alguna de estas opciones, sobre todo el tratamiento láser podra imponerse como alternativa válida a la resección transuretral y ocupar su lugar.

Por otro lado los varios métodos introducidos hace algún tiempo como la hipertermia, el HIFU, TUNA, TUMT, dilatación por balón, Stents uretrales etc. no han podido hasta el presente establecerce como tratamientos efectivos y su aplicación queda reducida a un grupo pequeño de pacientes por lo general con elevado riesgo quirúrgico.

En este trabajo fueron incluidos todos aquellos pacientes con indicación quirúrgica para el tratamiento de la HPB, con riesgo quirúrgico aceptable y un adenoma prostático grande (a partir de $50 \mathrm{~g}$ de tejido resecado) ${ }^{19,20}$.

En este estudio de 340 pacientes se analizaron retrospectivamente los resultados sobre la efectividad, complicaciones, morbi-mortalidad peri-operatoria, cambios sanguíneos de la hemoglobina, hematocrito y la concentración de hematíes después de la RTUP de baja presión hidráulica.

\section{PACIENTES, MATERIAL Y MÉTODO}

Se analizan los resultados de 340 pacientes con HPB sintomática que fueron operados con la RTUP de baja presión hidráulica entre agosto de 1999 y Junio del 2006 con adenoma grande.

El método se basa en el uso del trocar suprapúbico y un sistema de irrigación de baja presión hidrostática (entre 10 y $30 \mathrm{~cm} \mathrm{de} \mathrm{H}_{2} \mathrm{O}$ ) lo que evita la reabsorción de líquido de irrigación, independientemente de la duración de la RTUP (Fig. 1). 


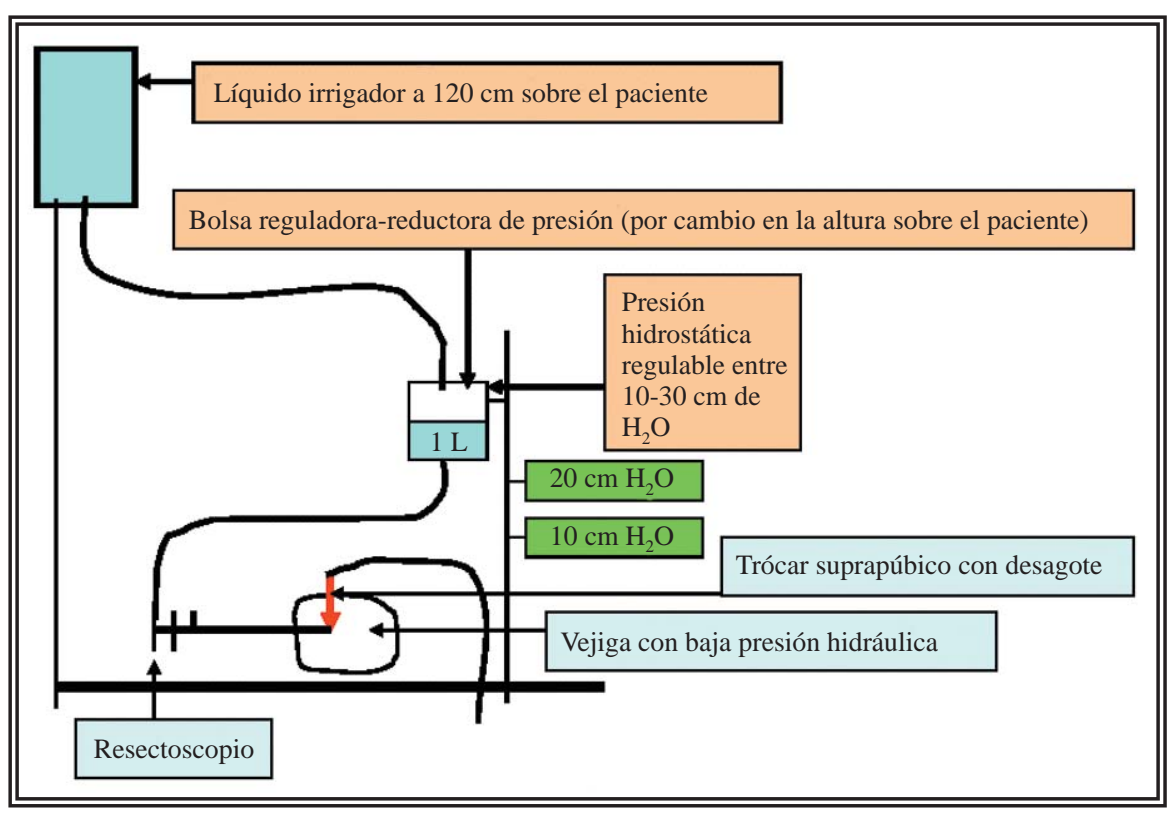

FIGURA 1. Esquema que demuestra el sistema de irrigación de baja presión hidráulica.

La baja presión intravesical constante, se obtiene a través de este método por medio de 2 dispositivos:

1. El líquido irrigador tiene una presión hidráulica entre 10 y hasta $30 \mathrm{~cm}$ de $\mathrm{H}_{2} \mathrm{O}$, regulable según la necesidad, y nunca mayor

2. Colocación de un trocar suprapúbico.

\section{RESULTADOS}

De los 340 pacientes operados, 20 (5,9\%) tenían al momento de la operación entre 50 y 59 años, 136 pacientes (40\%) entre 60 y 69 años, 156 pacientes $(45,9 \%)$ entre 70 y 79 años y 26 pacientes $(7,6 \%)$ entre 80 y 89 años (Fig. 2).

En 191 pacientes $(56,2 \%)$ se resecaron entre 50 y 69 g, en 99 pacientes $(29,2 \%)$ entre 70 y 99 $\mathrm{g}$ y en 50 pacientes (14,7\%) entre 100 y $160 \mathrm{~g}$ (Fig. 3) (Tabla 1).

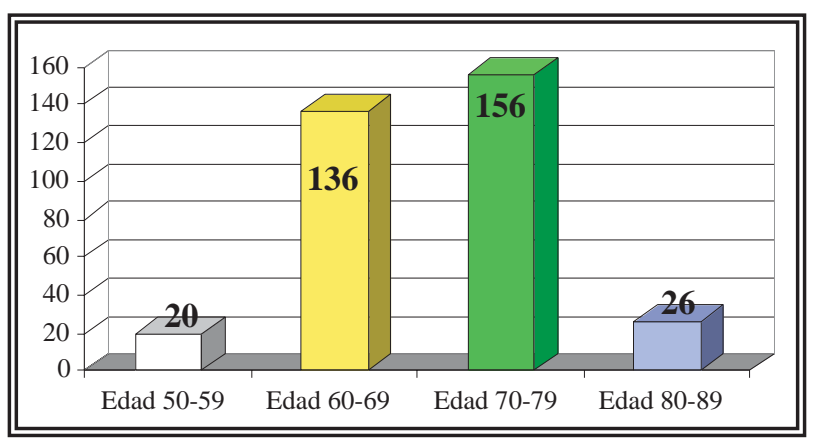

FIGURA 2. Distribución etaria de los 340 operados.
Control ecográfico mediante TRUS

Paciente con TRUS pre-op de $146 \mathrm{ml}$, RTUP de $120 \mathrm{~g}$ en 110 minutos. Control ecográfico por TRUS a los 9 días demuestra una celda prostática libre de coagulos y tejido adenomatoso residual (únicamente zona periférica) (Figs. 4 y 5).

Complicaciones intra y postoperatorias (Tabla 2).

Diagnósticos urológicos asociados (Tabla 3)

Espectro bacteriano detectado en los cultivos urinarios ( $\mathrm{Ta}$ bla 4).

Transfusión sanguínea (Fig. 6).

Valores de la hemoglobina pre y post operatoria en pacientes con y sin transfusión (Fig. 7).

Valores de la hemoblobina, hematocrito y eritrocitos en los pacientes con transfusión sanguínea, $n^{\circ}$ de pacientes 29 (8,5\%) (Tabla 5$)$.

Valores de la hemoglobina, hematocrito y eritrocitos en los pacientes sin transfusión sanguínea, $n^{\circ}$ de pacientes 311 (91,5\%) (Tabla 6).

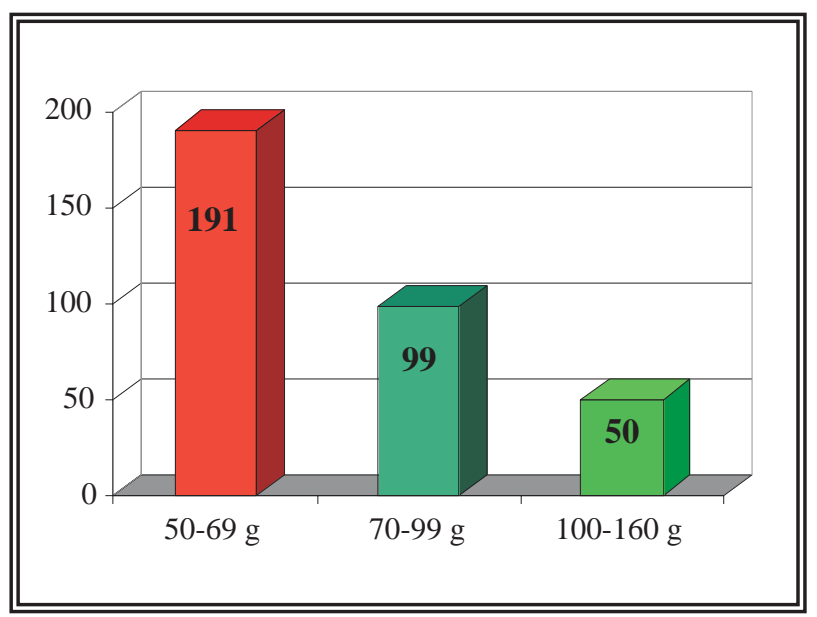

FIGURA 3. Distribución de los pacientes de acuerdo a peso resecado. 
Tabla 1

Resultados de los parámetros más significativos.

\begin{tabular}{lcc}
\hline & Promedio & Rango \\
\hline Edad & $69 \mathrm{años}$ & $51-89$ \\
TRUS & $110 \mathrm{ml}$ & $70-204$ \\
PSA & $7,94 \mathrm{ng} / \mathrm{ml}$ & $0,71-26,4$ \\
Capacidad vesical & $453 \mathrm{ml}$ & $150-1300$ \\
Tejido resecado & $81 \mathrm{~g}$ & $50-160$ \\
Tiempo de resección & $65 \mathrm{~min}$ & $35-155$ \\
Sondaje uretral & $1,7 \mathrm{días}$ & $1-8$ \\
Sondaje suprapúbico & $6,5 \mathrm{dias}$ & $5-15$ \\
Uroflujometría (preoperatoria) & $8,9 \mathrm{ml} / \mathrm{sec}$. & 6,512 \\
Uroflujometría (postoperatoria) & $19,7 \mathrm{ml} / \mathrm{sec}$. & $7-41,3$ \\
Residuo post miccional (preoperatorio) & $76 \mathrm{ml}$ & $20-400$ \\
Residuo post miccional (postoperatorio) & $25 \mathrm{ml}$ & $0-300$ \\
Estancia hospitalaria & $8 \mathrm{días}$ & $7-16$ \\
Riesgo quirúrgico bajo ASA I & $41 \%$ & \\
Riesgo quirúrgico medio ASA II & $52 \%$ & \\
Riesgo quirúrgico alto ASA III & $7 \%$ & \\
$\quad \begin{array}{l}\text { Anestesia espinal } \\
\text { Anestesia total }\end{array}$ & $337 \mathrm{pacientes}$ \\
\hline
\end{tabular}

\section{DISCUSIÓN}

El tamaño y peso de la próstata están en relación con la edad y la situación hormonal del paciente. El peso promedio en hombres entre 21 y 25 años oscila entre 17 y $28 \mathrm{~g}$, el largo es entre 3,1 a $4,2 \mathrm{~cm}$, el ancho de 3,5 a $5 \mathrm{~cm}$ y el diámetro antero posterior entre 1,7 y $2,3 \mathrm{~cm}^{21}$.

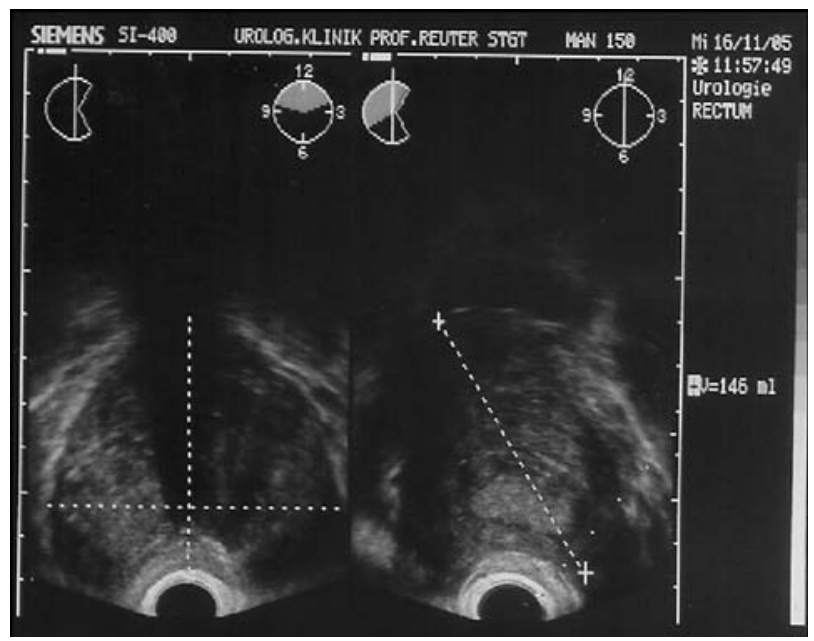

FIGURA 4. TRUS antes de la operación.
El parénquima glandular se divide en aprox. 30 a 50 lóbulos con 15 a 30 conductos excretores que desembocan en su mayoría en las cercanías del veru ${ }^{21}$.

Mc Neal divide en 1968 la próstata en tres zonas ${ }^{22-25}$ :

La zona periférica (ZP): En forma de herradura con la apertura ventral, representa el $70 \%$ del peso de una glandula sin hipertrofia.

La zona central (ZC): Representa hasta el $25 \%$ del peso glandular y posee forma cónica con la base en el cuello vesical y el vértice en el veru montanum y rodea el conducto eyaculador.

La zona transicional (ZT): Se encuentra peri-uretral y representa en una próstata normal solamente el 5\% de la masa de esta. Estudios histopatológicos demuestran que la HPB es un aumento del número de células y de esta forma un verdadero proceso hyperplásico. Es McNeal quien demuestra en sus estudios que los primitivos nódulos peri-uretrales se constituyen casi exclusivamente con tejido estromal y que su origen es la zona transicional. A la proliferación estromal inicial se le agrega posteriormente una epitelial, lo cual trasforma al proceso en un tumor benigno.

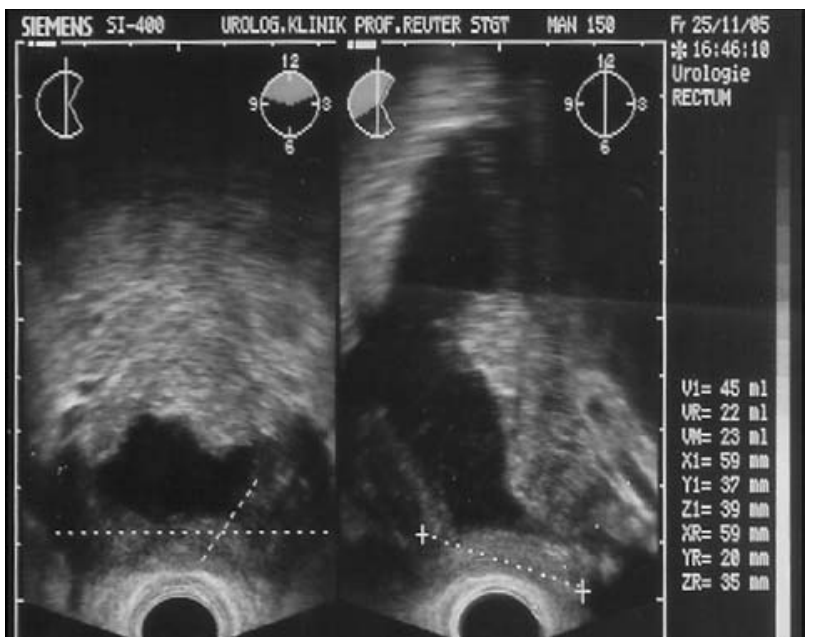

FIGURA 5. TRUS luego de la operación. 
Tabla 2

Complicaciones luego de RTUP en adenomas grandes

\begin{tabular}{lcc}
\hline & $\mathbf{n}^{\circ}$ pacientes & \% \\
\hline $\begin{array}{l}\text { Infección urinaria (bacterias > 100.000/ml) } \\
\text { Pérdida sanguínea que requirió transfusión } \\
\text { (hemoglobina < 8 gr/dl) }\end{array}$ & 95 & 27,9 \\
Retención urinaria postoperatoria & 18 & 5,5 \\
Perforación de la cápsula prostática & 14 & 4,1 \\
Taponamiento vesical por sangrado & 7 & 2 \\
Epididimitis & 3 & 0,9 \\
Complicaciones por punción con trócar & 3 & 0,9 \\
Vía falsa / lesión de la uretra bulbar & 2 & 0,6 \\
Hematoma escrotal grande por vasectomía & 1 & 0,3 \\
Lesiones del meato ureteral / resección accidental del meato & 0 & \\
Lesiones del pac. en sala op. /Nervio-Muscular- & & \\
decúbitos-quemaduras & 0 & \\
Incontinencia por lesión de esfínter & 0 & \\
Síndrome TUR & 0 & \\
Mortalidad & 0 &
\end{tabular}

Tabla 3

Diagnósticos asociados.

\begin{tabular}{lcc}
\hline & $\mathbf{n}^{\circ}$ pacientes & \% \\
\hline Estrechez de meato uretral/meatotomía & 28 & 8,2 \\
Vejiga hipotónica & 27 & 8 \\
Cáncer de próstata incidental & 25 & 7,3 \\
Cálculos vesicales/litotricia & 15 & 4,4 \\
Estrechez de uretra bulbar/uretrotomía & 11 & 3,2 \\
Hidronefrosis bilateral por obstrucción infravesical & 3 & 0,9 \\
Divertículo vesical grande/incisión & 3 & 0,9 \\
Cáncer de vejiga & 3 & 0,9
\end{tabular}

Tabla 4

Gérmenes detectados en orina.

\begin{tabular}{lcc}
\hline Germen />100.000 $\mathbf{~ u f c / m l}$ & $\begin{array}{c}\mathbf{n}^{\circ} \text { pacientes } \\
\text { afectados }\end{array}$ & \% \\
\hline Enterococo faecalis & 44 & 46,3 \\
Escherichia coli & 21 & 22,1 \\
Estafilococos/aureus/epidermidis/SMR & 10 & 10,5 \\
Pseudomonas aeruginosa & 5 & 5,2 \\
Proteus spp. & 4 & 4,2 \\
Klebsiellas spp. & 3 & 3,1 \\
Serratias spp. & 3 & 3,1 \\
Otros (acinetobacter/citrobacter/estreptococos/cándida spp. & 5 & 5,2 \\
\hline
\end{tabular}

La HPB es un tumor nodular benigno del tejido prostático con participación variable de sus componentes, glandulares, epiteliales, estromales o fibromusculares que llevan a un aumento del tamaño de la próstata ${ }^{22-25}$.

La RTUP representa en la actualidad el procedimento quirúrgico más extendido y el más utilizado en el tratamiento del adenoma prostático sintomático.

En un estudio sobre los métodos quirúrgicos empleados para la HPB sintomática efectuado por Treiber et al. en la Univ. de Munich en 2004 donde se incluyeron 137 clínicas urológicas en Alemania, se demostró que el $84,8 \%$ de los tratamientos fueron RTUP, el 9,6\% adenomectomías suprapúbicas, el 2,4\% fueron incisiones prostáticas transuretrales, el $1,3 \%$ tratamientos láser, el 0,6 \% electrovaporización, 0,7 \% fueron reseciones en medio salino, 0,4\% colocaron Stents. Varios procedimientos alternativos fueron directamente abandonados por sus escasos beneficios.

El sindrome de RTU que se caracteriza por presentar hipervolemia, hiponatremia, hipertensión, hemólisis, estado confusional, edema pulmonar, edema cerebral, etc. con depresión cardiocirculatoria y posteriormente fallo renal y oliguria, se produce en aquellos casos por reabsorción masiva de liquido de irrigación. 


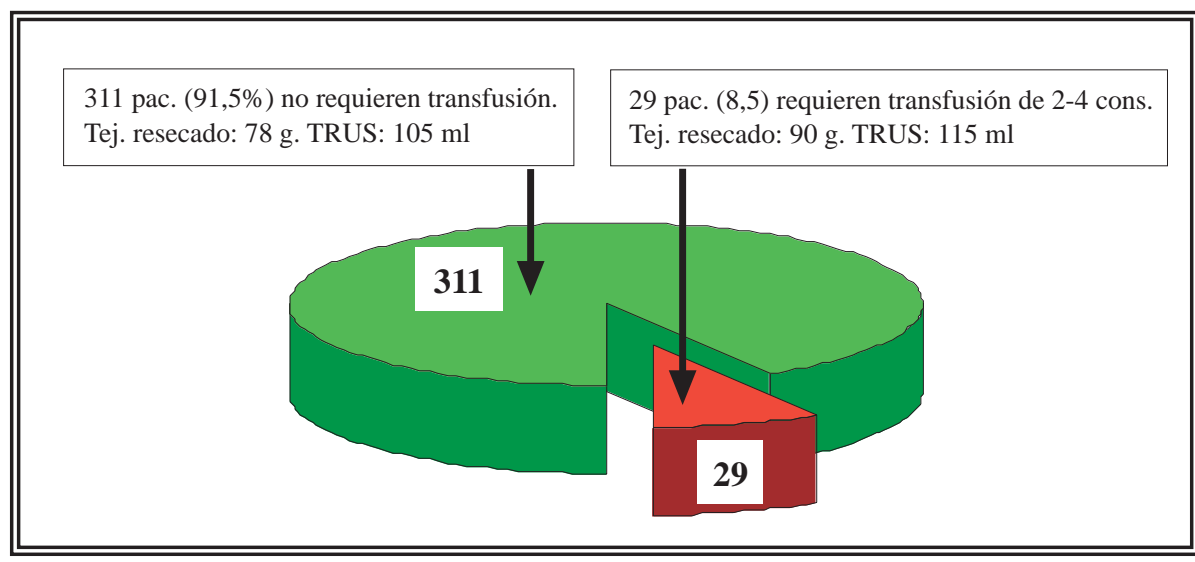

FIGURA 6. Distribución de los pacientes según transfusión sanguínea.

El origen del síndrome de RTU se presenta:

1. Al resecar en proximidades de la cápsula o perforarla, al abrir vasos venosos o al resecar los senos venosos periprostáticos.

2. Ante elevadas presiones intravesicales, mayores a $20 \mathrm{~cm}$ de $\mathrm{H}_{2} \mathrm{O}$, que equivale a la presión venosa pelviana en decúbito dorsal como cuando el bidón del líquido irrigador se encuentra a $100-120 \mathrm{~cm}$ sobre el paciente ${ }^{26}$.

3. En vejigas hipercontráctiles con disminuida compliance

En este estudio se pone de manifesto, las ventajas de combinar, el trócar suprapúbico y la baja presión del líquido irrigador entre 10 y $20 \mathrm{~cm}$ de $\mathrm{H}_{2} \mathrm{O}$, lo que garantiza durante toda la intervención, presiones intravesicales bajas, es decir por debajo de la presión venosa y de esta manera realizar resecciones de gran cantidad de tejido que no estan limitadas por el factor tiempo ya

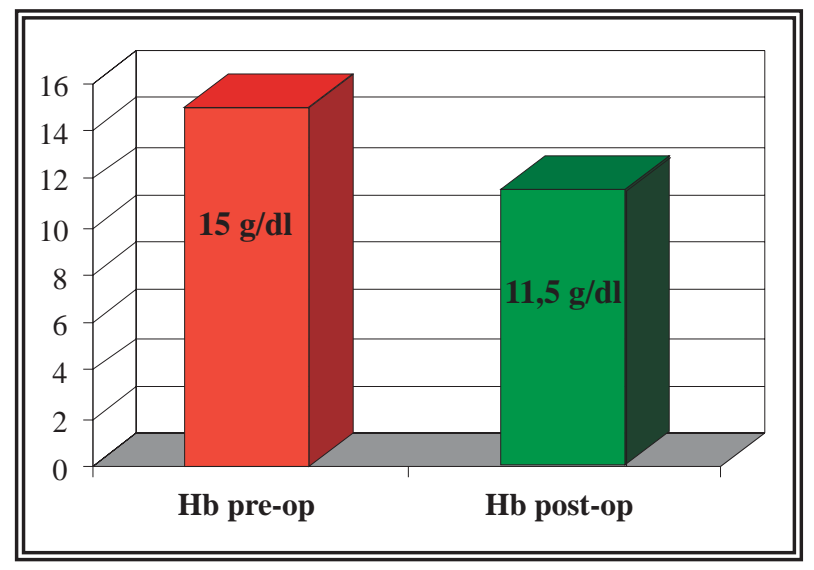

FIGURA 7. 311 pacientes sin transfusión. que no se produce la reabsorción indeseada de líquido irrigador. De esta forma tampoco es necesario interrumpir la intervención al producirse una perforación de la cápsula durante la RTU.

Paul Madsen de Madison, en Wisconsin fué uno de los primeros en reconocer la relación entre la presión hidrostática del líquido irrigador y la absorción del mismo $^{2}$.

Otra ventaja que representa la utilización del trócar suprapúbico es que permite, al finalizar la cirugía, la colocación de una sonda, permitiendose así la retirada precoz, en general a las 24 hs de la sonda transuretral y así evitar complicaciones (uretritis, infección urinaria, espasmos vesicales etc.). La sonda suprapúbica permite al cerrarla, el entrenamiento vesical después de la operación, efectuar una flujometría, también la medición exacta del residuo post-miccional y en caso de dificultades funcionar como "válvula de seguridad".

Debido a la seguridad que se obtiene resecando a bajas presiones intravesicales se relativizan los beneficios que habría al hacerlo con el sistema eléctrico bipolar. En un estudio randomizado donde se comparan los 2 métodos, efectuado por Singh $\mathrm{H}$. et al. se pone de manifiesto que la única ventaja del sitema bipolar es la falta de modifica-

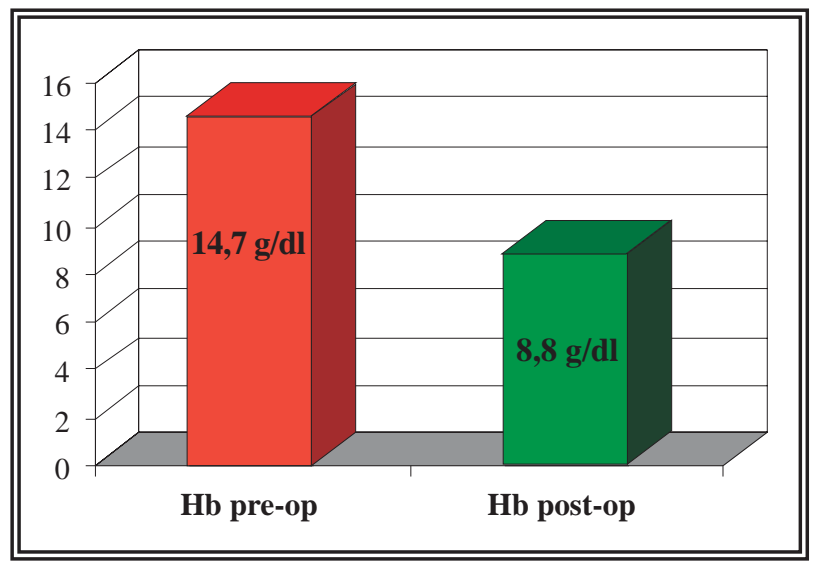

FIGURA 8. 29 pacientes con transfusión. 
Tabla 5

Valores sanguíneos en pacientes con transfusión

\begin{tabular}{lcccc}
\hline & & Valor & Rango & Diferencia \\
\hline Hemoglobina pre-op & $14,7 \mathrm{~g} / \mathrm{dl}$ & $12,4-17,1$ & \\
Hemoglobina & post-op & $8,8 \mathrm{~g} / \mathrm{dl}$ & $7,1-12,1$ & $(-) 5,9 \mathrm{~g} / \mathrm{dl}$ \\
Hematocrito & pre-op & $43,6 \%$ & $37,4-50 \%$ & \\
Hematocrito & post-op & $25,8 \%$ & $18,6-34,5 \%$ & $17,8 \%$ \\
Eritrocitos & pre-op & $4,8 \mathrm{~m} / \mathrm{ml}$ & $4,1-5,4 \mathrm{~m} / \mathrm{ml}$ & \\
Eritrocitos & post-op & $2,9 \mathrm{~m} / \mathrm{ml}$ & $2,3-3,5 \mathrm{~m} / \mathrm{ml}$ & $1,9 \mathrm{~m} / \mathrm{ml}$ \\
\hline
\end{tabular}

Tabla 6

Valores sanguíneos en pacientes sin transfusión.

\begin{tabular}{lcccc}
\hline & & Valor & Rango & Diferencia \\
\hline Hemoglobina & pre-op & $15 \mathrm{~g} / \mathrm{dl}$ & $12-19,3$ & \\
Hemoglobina & post-op & $11,5 \mathrm{~g} / \mathrm{dl}$ & $7,6-16,4$ & $(-) 3,5 \mathrm{~g} / \mathrm{dl}$ \\
Hematócrito & pre-op & $44,3 \%$ & $31,8-54,8 \%$ & \\
Hematócrito & post-op & $33,85 \%$ & $24,6-49,2 \%$ & $10,45 \%$ \\
Eritrocitos & pre-op & $4,88 \mathrm{~m} / \mathrm{ml}$ & $3,8-6,2 \mathrm{~m} / \mathrm{ml}$ & \\
Eritrocitos & post-op & $3,96 \mathrm{~m} / \mathrm{ml}$ & $2,7-4,5 \mathrm{~m} / \mathrm{ml}$ & $0,92 \mathrm{~m} / \mathrm{ml}$ \\
\hline
\end{tabular}

extraperitoneal. Edad de los pacientes 67,8 años, TRUS de 95,1 $\mathrm{ml}$, tiempo quirúrgico de 145 minutos, pérdida sanguínea de $192 \mathrm{ml}$, tejido resecado de 47,6 g, la estadía hospitalaria fue de 5,9 dias, sondaje uretral de 3 dias y 5 pacientes $(27,7 \%)$ presentan complicaciones postoperatorias $^{18}$.

Esto demuestra una falta de ventajas en casi todos los parámetros mencionados con respecto a la RTUP y hace evidente la cantidad de tejido residual remanente con la falta de desobstrucción completa.

En nuestra serie de 340 pacientes con RTUP se pudieron diagnosticar 25 adenocarcinomas incidentales de la próstata $(7,35 \%)$, los cuales tenian un PSA por de bajo de 4 ng/ml y una palpación prostática normal ${ }^{28}$.

ción del sodio sérico y la menor disuria post operatoria $^{16}$.

$\mathrm{Si}$ observamos los resultados publicados recientemente por distintos grupos que efectuaron adenomectomías abiertas como las de Millin o Freyer podemos apreciar un indice de transfusiones entre 16 y $20 \%$, con una pérdida sanguínea de $841 \mathrm{ml}$, reducción de la hemoglobina de $22 \mathrm{~g} / 1$, una estancia hospitalaria de 12 dias, una cateterización vesical de 6,5 dias un tiempo quirúrgico de $1,3 \mathrm{~h}$, una incontinencia urinaria de hasta $8 \%$, peso de tejido resecado 97,8 g 5 pacientes desarrollaron sintomas de obstrucción infravesical después de la operación ${ }^{15-27}$.

Otros autores como Muzzonigro et al. compararon los resultados de 113 pacientes operados con TURP a los cuales habían dividido en 2 grupos, el primero con próstatas de hasta $70 \mathrm{~g}$ (57 pac.) y el segundo grupo entre 70 y 150 g (56 pac.) y no encontró diferencias significativas de las complicaciones post-operatorias ${ }^{19}$.

Van Velthoven et al., de Bruselas (Bélgica), publica una serie de 18 pacientes operados con la técnica de adenomectomía prostática laparoscópica
Antes del alta se efectúa una TRUS con la intención de controlar la celda prostática y la efectividad del tratamiento. De esta forma se permite observar la falta de tejido adenomatoso residual que podría llevar en un futuro a recidivas del adenoma.

Si analizamos los datos obtenidos en una serie de 1.503 RTUP efectuadas en nuestro servicio, observamos que 37 pacientes debieron ser reoperados por presentar una obstrucción infravesical tiempo después del primer tratamiento, aproximadamente el 2,4\% del total ${ }^{29}$.

\section{CONCLUSIÓN}

La RTUP representa en la actualidad el procedimento quirúrgico más extendido y el más utilizado en el tratamiento del adenoma prostático sintomático.

La RTUP video-asistida de baja presión hidráulica, es un método efectivo y seguro en el tratamiento quirúrgico en adenomas prostáticos grandes de hasta $200 \mathrm{ml}$ medidos por la TRUS. Las complicaciones peri-operatorias severas son escasas, y comparables a las observadas en resecciones de menor volumen. 
Es importante destacar, las ventajas de asociar, el trocar suprapúbico y la baja presión hidráulica del líquido irrigador entre 10 y $20 \mathrm{~cm}$ de $\mathrm{H}_{2} \mathrm{O}$, lo que asegura durante toda la resección, presiones intravesicales bajas, es decir por debajo de la presión venosa periprostática y de esta manera realizar ablaciones de gran cantidad de tejido que no están limitadas por el factor tiempo ya que no se produce la reabsorción indeseada de líquido irrigador causantes del sindrome de RTU.

En 311 pacientes $(91,5 \%)$ no fue necesaria ninguna transfusión sanguínea. La estancia hospitalaria así como el sondaje uretral son menores en comparación con la adenomectomía abierta.

\section{REFERENCIAS}

1. Hauri D. Ein Blick in die Blase-der Mensch dahinter. Der Urologe A 2005;44(4):401-407.

2. Reuter MA et al. "History of Endoscopy". Band1: Geschichte der Endoskopie in der Antike, im Mittelalter und in 19.Jahrhundert. Band2: Der Weg zur Modernen Endoskopie. Band5: Geschichte der Transurethralen Prostatektomie (TURP). Karl Krämer Verlag Stuttgart + Zürich 1998.Editado en conjunto con el Museo Max Nitze.

3. Alschibaja M, May F, Treiber U, Paul R, Hartung R. Transurethrale Resektion bei benigner Prostatahyperplasie. Aktuelle Entwicklungen. Der Urologe A 2005;44(5): 499-504.

4. Litteljohn JO, Ghafar MA, Kang YM, Kaplan SA. Transurethral resection of the prostate: the new old standard. Curr Opin Urol. 2002 12(1):19-23.

5. Qiang W, Jianchen W, MacDonald R, Monga M, Wilt TJ. Antibiotic prophylaxis for transurethral prostatic resection in men with preoperative urine containing less than 100,000 bacteria per ml: a systematic review. The Journal of Urology 2005; 173(4):1175-1181.

6. Bachmann A, Wyler A, Ruszat R, Gasser T, Sulser T. Photoselektive KTP-laser vaporisation of the prostate. First experience after 50 procedures. J Endourol 2003 (Supp 1):A 188.

7. Bachmann A, Reich O, Wyler St, Ruszat R, Casella R, Gasser T, et al. Die 80-Watt-Kalium-Titanyl-Phosphat(KTP)-Laser-vaporisation der Prostata. Der Urologe A Okt 2004:43(10): 1262-1270.

8. Corica A, Marchiotto M, Verger-Kuhnke A, Anchelerguez R, Prats J. Urology Laser vaporisation of the prostate: experience in a selected group of patients. Congreso NorteAmericano de Urologia A.U.A. 1997.

9. Gurdal M, Tekin A, Yucebas E, Sengor F. Nd:YAG laser ablation plus transurethral resection for large prostates in high-risk patients. Urology 2003;62(5):914-917.

10. Hai MA, Malek RS. Photoselective vaporisation of the prostate: initial experience with a new $80 \mathrm{~W}$ KTP laser for the treatment of benign prostate hyperplasia. J Endourol. 2003;17(2):93-96.
11. Kumar SM. Photoselective vaporisation of the prostate: a volume reduction analysis in patients with lower urinary tract symptoms secondary to benign prostate hyperplasia and carcinoma of the prostate. J Urol. 2005;173(2):511513.

12. Kuntz RM, Lehrich K, Ahyai S. Transurethral holmium laser enucleation of the prostate compared with transvesical open prostatectomy: 18 month follow-up of a randomised trial. L Endourol. 2004;18(2):189-191.

13. Kuo RL, Kim SC, Lingeman JE, Paterson RF, Watkins SL, Simmons GR, Steele RE. Holmium laser enucleation of prostate (HoLEP): the Methodist Hospital experience with greater than 75 gram enucleations. J Urol. 2003;170(1): 149-152.

14. Reich O, Bachmann A, Schneede P, Zaak D, Sulser T, Hofstetter A.Experimental comparison of high power $(80$ W) potassium titanyl phosphate laser vaporisation and transurethral resection of the prostate. J Urol. 2004;171 (6Pt):2502-2504.

15. Shaheen A, Quinlan D. Feasibility of open simple prostatectomy with early vascular control. BJU Int. 2004; 93(3):349-352.

16. Singh H, Desai MR, Shrivastav P, Vani K Bipolar versus monopolar transurethral resection of prostate: randomized controlled study. J Endourol. 2005;19(3):333-338.

17. Van Melick HH, Van Venrooij GE, Eckhardt MD, Boon TA. A randomised controlled trial comparing transurethral resection of the prostate, contact laser prostatectomy and electrovaporisation in men with benign prostatic hyperplasia: urodynamic effects. J Urol. 2002;168(3):1058-1062.

18. Van Velthoven R, Peltier A, Laguna MP, Piechaud T. Laparoscopic extraperitoneal adenomectomy (Millin): pilot study on feasibility. Eur Urol. 2004;45(1):103-109.

19. Muzzonigro G, Milanese G, Minardi D, Yehia M, Galosi AB, Dellabella M. Safety and efficacy of transurethral resection of prostate glands up to $150 \mathrm{ml}$ : a prospective comparative study with 1 year with followup. J. Urol. 2004;172(2): 611-615.

20. Furuya S, Furuya R, Ogura H, Shimamura S, Araki T. Transurethral resection for prostatic adenoma larger than $100 \mathrm{ml}$-preoperative treatment with interstitial laser coagulation of the prostate plus chlormadinone acetate as a treatment maneuver for safer operations. Hinyokika Kiyo 2005;51(3):159-164.

21. Doerr, Seifert, Uehlinger. Compendio de Anatomia Patologica, Tomo 21. Spezielle pathologische AnatomieBand 21(Hoden.Prostata.Samenblasen). Springer-Verlag Berlin-Heidelberg 1991-Germany.

22. Mc Neal JE. Regional morphologie and pathologie of the prostata. Am J Clin.Pathol. 1968;49:347-357.

23. Mc Neal JE. Origen and evolution of benign prostata enlargement. Invest. Urol. 1978;15:340-345.

24. Mc Neal JE: The prostate gland: Morphologie and pathobiologie. Monogr Urol. 1988;9:3-33.

25. Mc Neal JE: Prostate. Histologie for Phatologists, second edition. 1997.

26. Bliem F, Lamche M, Janda R, Ilias W, Schramek P. Blutverlust und Einschwemung bei TURP vs. TURVP unter Niederdruck-bzw. Hochdruckbedingungen. Der Urologe A 2003.42(11): 1477-1484.

27. Bapat RD, Relekar RG, Pandit SR, Dandekar NP. Comparative study between modified Freyer's prostatectomy, classical Freyer's prostatectomy and Millin's prostatectomy. J Postgrad Med. 1991;37(3):144-147. 
28. Kitamura H, Masumori N, Tanuma Y, Yanase M, Itoh N, Takahashi A, et al. Does transurethral resection of the prostate facilitate detection of clinically significant prostate cancer that is missed with systematic sextant and transition zone biopsies?. Int J Urol. 2002;9(2):9599.

29. Shimizu Y, Hiraoka Y, Iwamoto K, Takahashi H, Abe H. Measurement of residual adenoma after transurethral resection of the prostate by transurethral enucleation technique. Urol Int. 2005;74(2):102-107.
30. Zamann A.P., Zajaczkowski T. Julius Bruck (1840-1902) Sein Beitrag zur Weiterentwicklung der Endoskopie. Der Urologe B 2002.42:45-39.

Dr. A.B. Verger Kuhnke

Hackstraße 61

70190 Stuttgart (Baden-Württemberg) Germany

E-mail: andresbverger@aol.com

(Trabajo recibido el 5 de julio de 2006)

\section{COMENTARIO EDITORIAL}

El artículo analiza los resultados obtenidos mediante Resección Transuretral a a baja presión en una serie significativa de pacientes afectos inicialmente de Hiperplasia Benigna de Próstata (HBP) de gran tamaño, con cifras de resección siempre superiores a los 50 gramos sin ser excepcional (15\%) los valores entre 100 y $160 \mathrm{~g}$.

Los autores deben ser felicitados por los excelentes resultados clínicos obtenidos y que se exponene en el citado trabajo con una sistemática y rigor científico impecables. A señalar dos comentarios puntuales y muy breves sobre la resección bipolar y la adenomectomía laparoscópica que se hacen en el artículo (citas 16 y 18). Ambos temas, junto al papel del láser en esta patología, son potencialmente controvertidos.

La única objeción, ni siquiera crítica pues lamentablemente es un hecho casi habitual en la literatura urológica, que no puede dejar de señalarse al menos en el marco de la Revista en que se publica el artículo, es la ausencia en la revisión histórica y bibliografía de apoyo de los ingentes trabajos e investigación sobre esta patología de Salvador Gil Vernet ${ }^{1}$, ciertamente muy anteriores a los de Mc Neal (referencias $22,23,24$ y 25 del trabajo) y en los que ya señalaban con exquisito rigor la mayoría de los hallazgos popularizador a partir de las referencias que se citan.

\section{REFERENCIAS}

1. Gil Vernet S. Patología Urogenital, Tomo II, Vol. I: Biología y Patología de la próstata. Madrid, Paz Montalvo, 1953.

Fdo.: Dr. A. Ipiens Aznar Jefe del Servicio de Urología. Hospital de Navarra. 